\title{
An Optimized Ant Colony Algorithm to Reduce the Impact of Flooding using Clustering in Ad Hoc Network
}

\author{
Rajdeep Singh Chauhan \\ M-Tech (C.S.E.) \\ GNDEC Ludhiana
}

\author{
Jasbir Singh Saini \\ Associate Professor \\ GNDEC Ludhiana
}

\author{
Harwinder Singh Sohal \\ Assistant Professor \\ LLRIET Moga
}

\begin{abstract}
Flooding is a phenomenon used in adhoc networks to get the information about the other nodes in the network. Whenever the topology in adhoc network changes the node has to perform the flooding technique to get the shortest path for neighborhood nodes. By using this technique, the information seeking packets from the source nodes are sent to all the other nodes in neighbor to get the information regarding the different routing paths and the routing distances for the other nodes. Ant algorithm is one of the best approaches to get the shortest path of the neighborhood nodes. The shortest path is get by using flooding technique in ant algorithm but there is a problem, when the changes in the network are frequent \& dynamic, for each \& every change, the node has to perform flooding to get the shortest path which give rise to the problem of congestion \& decreases the throughput. Both of the problems results in the increase of end to end delay. This motivates the need of clustering technique to handle such problems. The present work describes that the whole network nodes are divided into clusters. So rather then using the flooding technique for all the neighbor nodes, the flooding is used only among the clusters which reduces the network congestion and results in the increase of throughput \& decrease in the end to end delay.
\end{abstract}

\section{Keywords}

Flooding, Ant, Clusters.

\section{INTRODUCTION}

\subsection{Wireless Ad-hoc network}

A wireless Ad-hoc network is a wireless network having no particular centralized node. The name ad-hoc in referred because the realization of the network is not dependent upon the previous infrastructure. In this network each node gives its contribution in the transmission of data by forwarding the data to the other nodes and getting the data from the other nodes. All the nodes in this kind of network have same priority which means all the nodes have to follow the same set of rules for transmitting the data. The nodes in ad-hoc network are dynamic nodes i.e. these nodes changes their position by the time. So to determine the optimized route for transmitting the data among the nodes of the ad-hoc network is a great challenge because of the dynamic changes in the topology which occur very frequently [1]. Wireless ad-hoc network is an autonomous system of mobile nodes connected by wireless links; each node operates as an end system and a router for all other nodes in the network. Nodes in mobile ad-hoc network are free to move and organize themselves in an arbitrary fashion. Each node is free to roam while communicating with others. The path between each pair of the nodes may have multiple links and the radio between them can be heterogeneous. This allows an association of various links to be a part of the same network [2].

\subsection{Flooding}

Flooding is a phenomenon which is used by the nodes of adhoc network to get the routing information about the neighborhood nodes in the network. Flooding starts with the one node which sends the flooding packet to the other neighbor nodes to get the routing information about them. The flooding packet received by the neighbor nodes again send the same type of packet to their neighbor nodes and also reply their status to the previous node. This process keeps on repeated by all the nodes till the routing tables are not completed for each node. These routing tables further help the nodes to get the information about the factors which are used in networking for e.g. routing distance, routing path etc.

\subsection{Ant's Colony Algorithm}

The frequent changes in the topology of the ad-hoc network make it difficult to find the shortest path between the sender and the receiver because the location of the nodes keeps on changing. So to find the shortest path between the nodes of the network to transfer the data is a big challenge, which inspires to use some efficient algorithm in the dynamic environment. However Ant's algorithm overcomes this problem by using the ant's phenomena to find the shortest path. When an ant moves on the path to search for food it follows the chemical known as pheromone which is secreted by the other ant on the way of food and when it reaches at a point where there are more the one ways to the food then the ant take the decision to follow the path based upon the intensity of the pheromone secreted by the other ants. It follows the path having more intensity of pheromone [3].This leads all the ants to find the shortest path between their nests and the food as the time passes away. The pheromone on the branches of the shortest path to the food grows faster than pheromone on other branches. If the obstacle is being put on the way of ant's nest $\&$ the food having two branches of the path to the food, one is shorter than the other. The ants deposit pheromone while moving. The ant which has chosen the shorter path will reach first to the food, pick up and then return back to the nest. It will repeat the same phenomena again and again. When other ants reach the food, they will also follow shortest path since this path has more pheromone [4].

\section{LITERATURE SURVEY}

Gabber E. et. al (2004) [5] discussed a new intra-domain IP routing algorithm called TRAIL BLAZER (TB) that alleviates network congestion by local decisions based on latency measurements collected by scout packets. TB is a member of a class of traffic-aware routing algorithms based on the behavior of ants. TB maintains in every router a probability table that controls the selection of outgoing links for a given destination. This table is modified by passing scout packets. Some scout packets follow existing high probability paths, and other scout packets explore new paths by making random "mistakes" in order to find detours around congested areas of 
the network. Scout packets do not have global knowledge of the network topology. Rather, they are influenced by probability trails left by previous scout packets, and leave a trail of updated probability information. Even though TB may cause packet reordering which may affect the congesting control mechanisms of TCP, TCP traffic sent over TB has a similar bandwidth to shortest-path routing.

Chen G., et al. (2006) [6] discussed that the routing in wireless sensor networks is very challenging due to their inherent characteristics of large scale, no global identification, dynamic topology and very limited power, memory and computational capacities for each sensor. Recent researches on WSNs routing protocol has proved that data centric technologies are needed for performing in network aggregation of date to yield energy-efficient dissemination. Optimization (ACO) algorithms have been introduced to design the data centric routing protocol \& have got many achievements, but still have some shortcomings blocking their further applications in the large scale WSN's to overcome the flaws of conventional ant based data centric routing algorithm, they proposed an improve protocol by adding a new type of ant, search ant, to supply prior information to the following ants.

LI B., et al. (2009) [7] discussed new dynamic spectrum environment in cognitive radio networks necessities novel routing protocols which should be spectrum-aware, selfconfigured, high-adaptive and robust to match the dynamic nature of Cognitive Radio Network (CRN) and exploit the available spectrum efficiently. In this paper, the author presents ASAR, which is a biologically inspired routing solution for CRN. In the routing algorithm, paths are discovered, observed and learned by guided ants communicating in an indirect way. F-ants are used to exploit spectrum feasible paths to the destinations and B-ants are used to collect information about the network and update routing table in the nodes.

Bandyopadhyay M., et al. (2010) [8] elaborates Ant colony optimization (ACO) is a stochastic approach for solving combinatorial optimization problems like routing in computer networks. Zone based routing algorithms is build on the concept of individual node's position for routing of packets in mobile ad-hoc networks. Here in this paper they have used Zone based ANT colony using Clustering which assures to find shortest route using the DIR principle together with minimum overhead for route discovery and mobility management. Unlike other Zone based approach, in clustering it is not required to consider zone related information of each node while finding shortest path. Here, it is being proposed a new routing algorithm for mobile ad hoc network by combining the concept of Ant Colony approach and Zone based routing approach using clustering to get shortest path.

Magyare E.A., et al. (2010) [9] discussed a novel routing algorithm called Bees Ants algorithm. This algorithm is a combination of Ant colony based Routing Algorithm (ARA) and Bee Hive based Routing Algorithm. The proposed routing algorithm depends on splitting the network into two parts; one is a fixed network and the other is a mobile ad hoc network (MANET), then applying the Ant colony based Routing Algorithm on the mobile part and the Bee Hive based Routing Algorithm on the fixed one. After comparing the proposed algorithm with the ARA algorithm, it shows promising results in terms of propagation delay, queue delay, and number of hops.

\section{PROBLEM FORMULATION}

The problem with Flooding Technique is described as follows:

As it is known that the ant colony algorithm is used to find the shortest path between the nodes of network but when this algorithm is used on wireless adhoc network it makes the algorithm to work much frequently because of the dynamic changes in the location of the nodes. So when there is a change in topology of the network each time the flooding technique is to be used to find the shortest path, which consumes most of the bandwidth of the network and result in decreased in throughput \& increased end-to-end delay.

\subsection{Objectives}

The clustering technique is used to reduce the effect of flooding to achieve the following objectives:

1. To achieve improved Throughput.

2. To decrease End to End Delay.

\section{PRESENT WORK}

The dynamic change in topology leads to network traffic as discussed earlier. This problem can be optimized by dividing the network into clusters. All the nodes of wireless Ad-hoc network are divided according to the cluster i.e. C1, C2, $\mathrm{C} 3 \ldots \mathrm{Cn}$. Each cluster is having a cluster address. So at the time of the changes in the topology, the flooding is done among the clusters having a specific cluster address instead of all nodes, which reduces the traffic over the network. In the present work the scenario consist of 50 nodes in the network which is divided into 5 clusters according to the geographical area. Whenever a node goes from one cluster to another cluster the cluster information is updated. Each cluster of multiple nodes having unique address which represents that particular cluster, whenever there is a need to send the data to any node the data is sent to the cluster address. The optimized algorithm is divided into various steps. The steps for the optimization of ant algorithm using clustering are shown in the figure 4.1 .

The algorithm starts with the collection of the status of all the nodes which are contained in the network. After that the whole network is divided into the clusters based on their geographical area. Each cluster is given an address, so that whenever there is need to send the data, it is sent to the cluster address and the required node for which the data is sent receives the data. In algorithm, it is continuously checked that weather the topology is changed in the network or not. Whenever there is any change in the topology, the flooding technique is performed but rather then in the old algorithm, in which the flooding was performed between all the nodes, in this optimized algorithm the flooding is performed in the clusters only. It reduces the load of flooding packets which were sent in earlier algorithm to just 5 clusters instead of 50 nodes in old algorithm. According to the flooding packets sent and received by the various nodes of the clusters the routing table of the cluster is updated and data transmission is performed according to the routing table. 


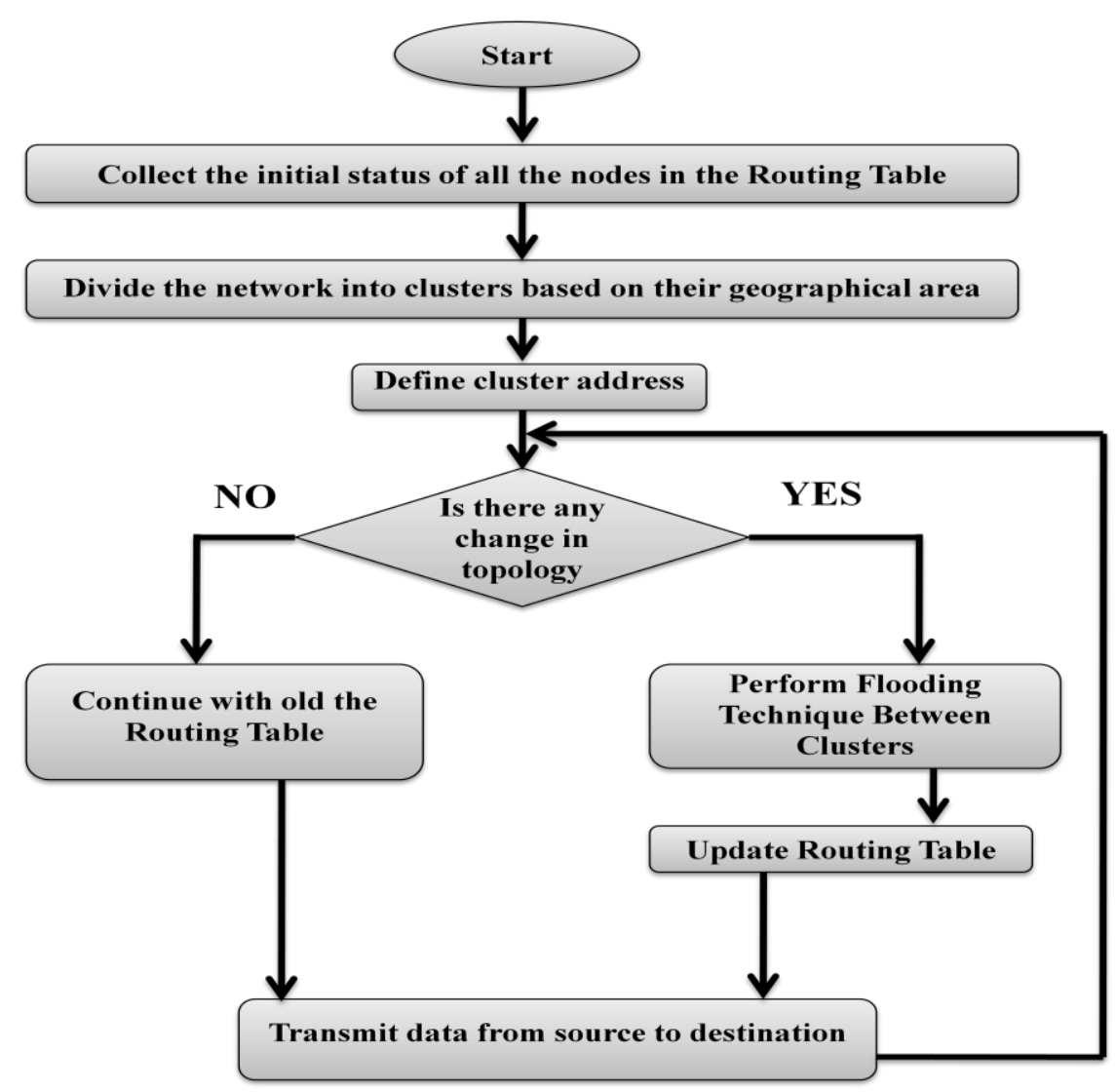

Figure: 4.1 Flow Chart describing the steps for the optimization of ant algorithm using clustering.

\section{SIMULATION RESULTS}

\subsection{Simulation Environment}

The simulation experiment is carried out in LINUX (REDHAT version 5). The detailed simulation model is based on network simulator-2 (ver-2.34), is used in the evaluation. The NS instructions can be used to define the topology structure of the network and the motion mode of the nodes, to configure the service source and the receiver, to create the statistical data track file and so on.

\subsection{Simulation Parameters}

Table 1: Simulation parameters

\begin{tabular}{|l|l|}
\hline \multicolumn{1}{|c|}{ Parameter } & \multicolumn{1}{c|}{ Value } \\
\hline Environment Size & $500 \times 500$ \\
\hline Packet Size & 512 Byte \\
\hline Traffic Type & CBR \\
\hline Bandwidth & $40 \mathrm{MHZ}$ \\
\hline MAC Protocol & IEEE 802.11 \\
\hline Antenna Type & Omni Directional \\
\hline
\end{tabular}

\subsection{Traffic Model}

The continues bit rate (CBR) is used in the simulator. The source nodes as well as the destination nodes were spread over the region of network randomly. The scenario of 50 nodes which are divided into 5 clusters initially is developed.
The following figure.5.1 and figure 5.2 shows the scenario based on old algorithm as well as new optimized algorithm.

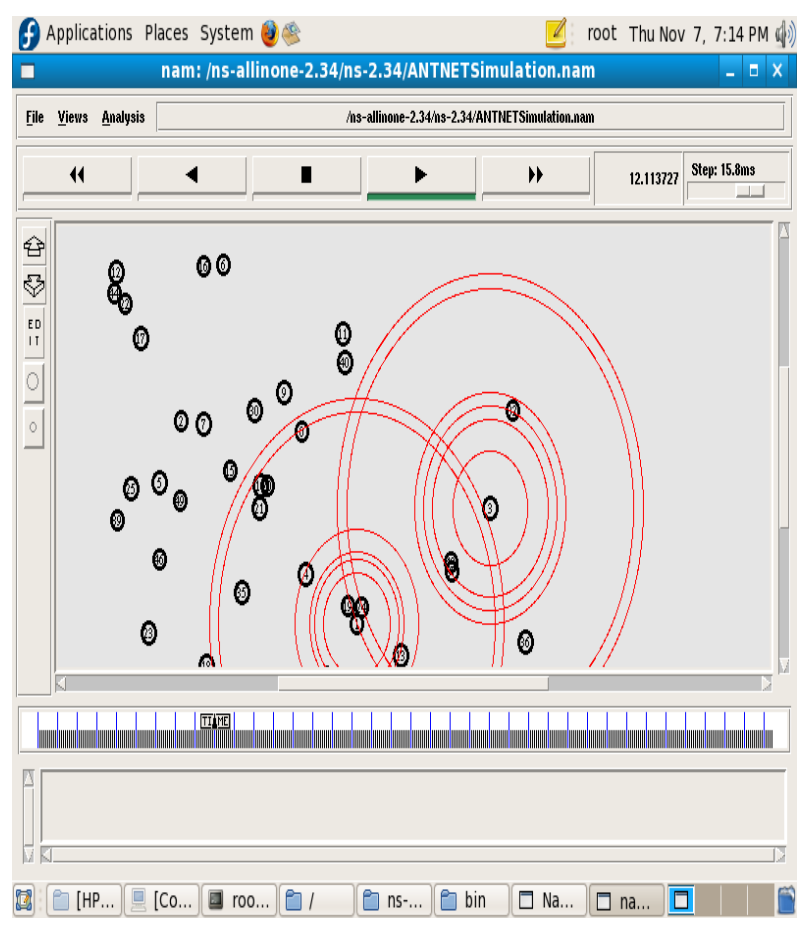

Figure 5.1 Old Ant Colony Algorithm without Clustering 


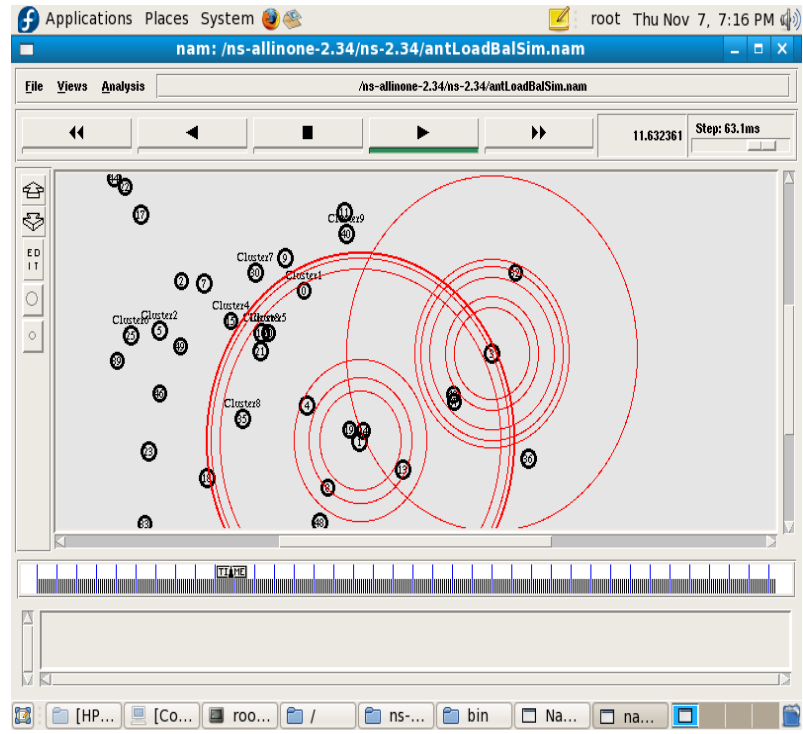

Figure: 5.2 Optimized Ant Colony Algorithm Scenario with Clusters

\subsection{Performance Results}

The simulation is performed on both the scenarios i.e. old ant colony algorithm as well as the optimized ant colony algorithm keeping same movements models. The End-To-End Delay and Throughput is measured as performance metrics for both the algorithms.

\subsubsection{End To End Delay}

The end-to-end delay is a parameter is the total time taken by a packet to reach from source node to the destination node. The performance of the network can be evaluated by finding the end-to-end delay. As the value of this parameter is less this indicates that the packet will take less time to reach its destination. Then the destination node has to wait less for the packets which improves the efficiency of the network. In the fig below the eeAnt.xgr and eeAntLoad.xgr are the graphs for old ant colony algorithm and naval optimized ant colony algorithm respectively. The end-to-end delay parameter is less in the new optimized ant colony algorithm which shows the efficiency of this optimized algorithm.

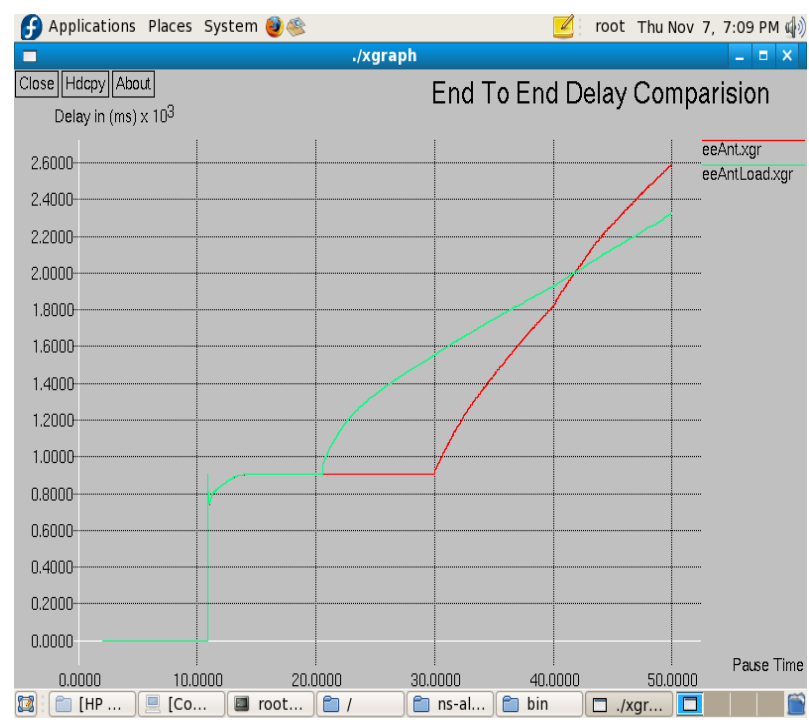

Figure: 5.3: Comparison of Ant \& Optimized Ant Algorithm in terms of end-to-end delay

\subsubsection{Throughput}

It is the fundamental parameter to check the performance on the basis of data transfer rate by the network nodes. It is calculated as average rate of successful message delivery over a communication channel. The throughput is usually measured in bits per second (bit/s or bps), and sometimes in as the throughput is increased which indicate the increased the data transmission of the network in a scenario data packets per second or data packets per time slot.

The antTp.xgr and antTP.xgr shows the throughput of old ant colony algorithm as well as new ant colony algorithm resp. because it take some time to divide the network in to cluster so initial part of the pause time shows the decreased throughput, but when the clusters have been made the throughput of the network increases significantly which shows the enhancement of the performance of new algorithm.

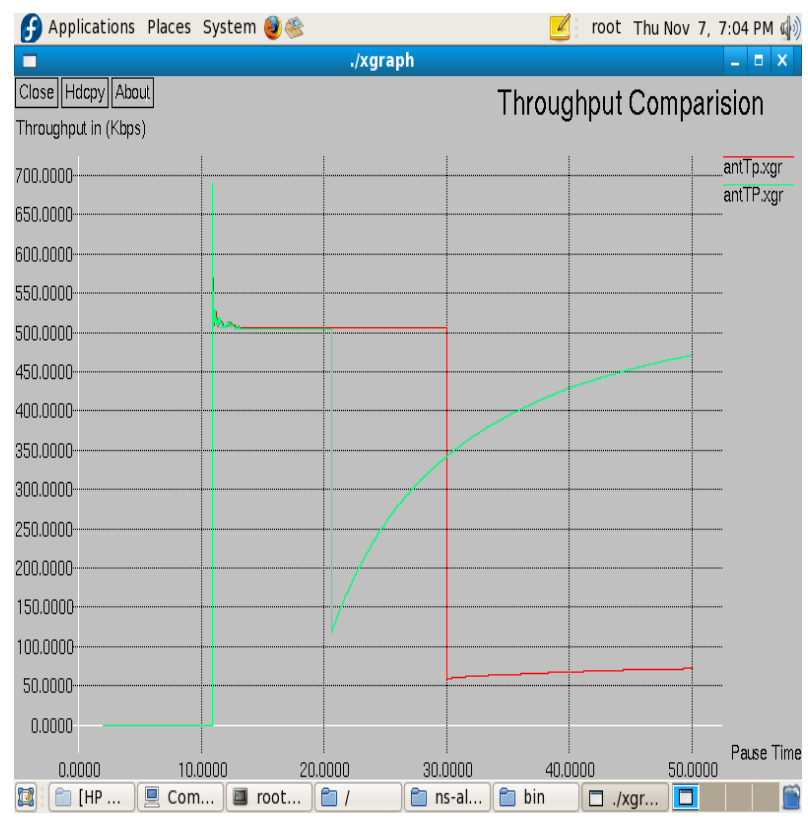

Figure: 5.4: Comparison of Ant \& Optimized Ant Algorithm in terms of Throughput

\section{CONCLUSION \& FUTURE WORK}

It can be concluded from the above results that the novel optimized algorithm works more efficiently in terms of throughput as well as end-to-end delay. The experimental results show a sufficient increased throughput and decrease in the end-to-end delay in the network which improves the efficiency of the old algorithm as well as the network.

In future the novel optimized ant colony algorithm can be used on larger network in which the concept of multi clustering (clusters with in the clusters) can be used to handle the network traffic. The parameters like energy conservation and packet drop ratio are still to find. The future work comprises of find the parameters and also to use this algorithm on larger network in more optimized way.

\section{REFERENCES}

[1] D. Kim, J. Garcia and K. Obraczka, "Routing Mechanisms for Mobile Ad Hoc Networks based on the Energy Drain Rate", IEEE Transactions on Mobile Computing. Vol 2, no 2, 2003, pp.161-173. 
[2] Wenjing Guo, Wei Zhang, Gang Lu "A Comprehensive Routing Protocol in Wireless Sensor Network Based on Ant Colony Algorithm" 2010 Second International Conference on Networks Security, Wireless

[3] Yanhua Li, Zhi-Li Zhang, and Daniel Boley "From Shortest-path to All-path: The Routing Continuum Theory and its applications" IEEE TRANSACTIONS ON PARALLEL AND DISTRIBUTED SYSTEMS, VOL. X, NO. X, JANUARY 201X, PP 1-11.

[4] Hussein O., Saadawi T. "Ant Routing Algorithm for Mobile Ad-hoc Networks (ARAMA)*”.

[5] Gabber E., Smith M. A. (2004) "Trail Blazer: A Routing Algorithm Inspired By Ants", IEEE International Conference on Network Protocols (ICNP'04).

[6] Chen G., Guo T., Wang W., Zhao T. (2006), "An Improved Ant-Based Routing Protocol In Wireless Sensor Networks"1-4244-0429-0-/06(IEEE 2006).
Communications and trusted Computing, 2010 Second International Conference on Network Security, Wireless Communications and Trusted Computing, PP 41-44.

[7] LI B., LI D., LI H. (2009), “ASAR: Ant-Based Spectrum Aware Routing For Congnitive Radio Networks" 9781-4244-5668-0/9(IEEE 2009).

[8] Bandyopadhyay M., Baumik P (2010) "Zone Based Ant Colony Routing In Mobile Ad-hoc Network" Copyright Clearance Centre (CCC) 978-1-4244-5489-1/10(IEEE 2010).

[9] Maghayreh E.A., Al-Haija S.A., Alkhateeb F., Alijawarneh A. (2010) "Bees_Ant Based Routing Algorithm”978-0-7695-3973-7/10(IEEE 